\title{
TWO WEIGHT FUNCTION NORM INEQUALITIES FOR THE POISSON INTEGRAL
}

\author{
BY \\ BENJAMIN MUCKENHOUPT $\left({ }^{1}\right)$
}

ABSTRACT. Let $f(x)$ denote a complex valued function with period $2 \pi$, let

$$
P_{r}(f, x)=\frac{1}{2 \pi} \int_{-\pi}^{\pi} \frac{\left(1-r^{2}\right) f(y) d y}{1-2 r \cos (x-y)+r^{2}}
$$

be the Poisson integral of $f(x)$ and let $|I|$ denote the length of an interval $I$. For $1<p<\infty$ and nonnegative $U(x)$ and $V(x)$ with period $2 \pi$ it is shown that there is a $C$, independent of $f$, such that

$$
\sup _{0 \leqslant r<1} \int_{-\pi}^{\pi}\left|P_{r}(f, x)\right|^{p} U(x) d x<C \int_{-\pi}^{\pi}|f(x)|^{p} V(x) d x
$$

if and only if there is a $B$ such that for all intervals $I$

$$
\left[\frac{1}{|I|} \int_{I} U(x) d x\right]\left[\frac{1}{|I|} \int_{I}[V(x)]^{-1 /(p-1)} d x\right]^{p-1 .}<B \text {. }
$$

Similar results are obtained for the nonperiodic case and in the case where $U(x) d x$ and $V(x) d x$ are replaced by measures.

1. Introduction. In [4] Rosenblum showed that if $1 \leqslant p<\infty, 0 \leqslant r<1$, $f(x)$ has period $2 \pi$ and $\mu$ is a finite Borel measure with period $2 \pi$, then there is a $C$, independent of $r$ and $f$, such that

$$
\int_{-\pi}^{\pi}\left|P_{r}(f, x)\right|^{p} d \mu(x) \leqslant C \int_{-\pi}^{\pi}|f(x)|^{p} d \mu(x)
$$

if and only if $d \mu(x)=V(x) d x$ is absolutely continuous and there is a constant $K$, independent of $x$ and $h$, such that

$$
\frac{1}{h} \int_{x-h}^{x+h}\left[\frac{1}{h V(t)} \int_{t-h}^{t+h} V(s) d s\right]^{1 /(p-1)} d t \leqslant K
$$

for all $h>0$ and all $x$. It is easy to see that a necessary and sufficient condition for (1.2) to hold is that there is a $B$ such that for every interval $I$

$$
\left[\frac{1}{|I|} \int_{I} V(x) d x\right]\left[\frac{1}{|I|} \int_{I}[V(x)]^{-1 /(p-1)} d x\right]^{p-1} \leqslant B
$$

Received by the editors July $23,1974$.

AMS (MOS) subject classifications (1970). Primary 26A86, 44A15; Secondary 40G10, $42 \mathrm{~A} 24$.

(1)Supported in part by NSF grant GP 38540. 
this follows from the fact that the left side of (1.2) is bounded below by

and above by

$$
\frac{1}{h} \int_{x-h / 2}^{x+h / 2}\left[\frac{1}{h V(t)} \int_{x-h / 2}^{x+h / 2} V(s) d s\right]^{1 /(p-1)} d t
$$

$$
\frac{1}{h} \int_{x-2 h}^{x+2 h}\left[\frac{1}{h V(t)} \int_{x-2 h}^{x+2 h} V(s) d s\right]^{1 /(p-1)} d t .
$$

Rosenblum also considered (1.1) when $p=1$; he proved the same result with (1.2) replaced by

$$
\frac{1}{h} \int_{x-h}^{x+h} V(t) d t \leqslant K V(x) .
$$

The purpose of this paper is to give a simpler proof than Rosenblum's of a more general result. Specifically, the following will be proved in $\S \S 2$ and 3 .

THEOREM 1. If $1 \leqslant p<\infty, 0 \leqslant r<1, \mu$ and $\nu$ are Borel measures of period $2 \pi$ and $f(x)$ has period $2 \pi$, then there is $a C$, independent of $f$ and $r$, such that

$$
\int_{-\pi}^{\pi}\left|P_{r}(f, x)\right|^{p} d \mu(x) \leqslant C \int_{-\pi}^{\pi}|f(x)|^{p} d \nu(x)
$$

if and only if for every interval $I$

$$
\left[\frac{\mu(I)}{|I|}\right]\left[\frac{1}{|I|} \int_{I}\left[\frac{d v_{a}(x)}{d x}\right]^{-1 /(p-1)} d x\right]^{p-1} \leqslant B,
$$

where $B$ is independent of $I$ and $\nu_{a}$ denotes the absolutely continuous part of $\nu$.

In Theorem 1 and throughout this paper

$$
\left[\frac{1}{|I|} \int_{I}\left[\frac{d v_{a}(x)}{d x}\right]^{-1 /(p-1)} d x\right]^{p-1}
$$

is to be interpreted as ess $\sup _{x \in I}\left[d v_{a}(x) / d x\right]^{-1}$ if $p=1$ and $0 \cdot \infty$ is to be interpreted as 0 .

The nonperiodic version of Theorem 1 will follow from the same reasoning; this is stated as Theorem 2 in $\S 4$. The fact that for nonnegative $U(x)$ and $V(x)$

$$
\sup _{0 \leqslant r<1} \int_{-\pi}^{\pi}\left|P_{r}(f, x)\right|^{p} U(x) d x \leqslant C \int_{-\pi}^{\pi}|f(x)|^{p} V(x) d x
$$

holds if and only if for every interval $I$

$$
\left[\frac{1}{|I|} \int_{I} U(x) d x\right]\left[\frac{1}{|I|} \int_{I}[V(x)]^{-1 /(p-1)} d x\right]^{p-1} \leqslant B
$$

is an immediate corollary of Theorem 1.

In the light of recent results concerning other operators, Theorem 1 and this 
corollary are not as natural an extension of Rosenblum's result as they may seem. For example, if $1<p<\infty, U(x)$ and $V(x)$ are nonnegative and $U(x)=V(x)$, then there is a $C$, independent of $f$, such that

$$
\int_{-\pi}^{\pi}\left(\sup _{0 \leqslant r<1}\left|P_{r}(f, x)\right|\right)^{p} U(x) d x \leqslant C \int_{-\pi}^{\pi}|f(x)|^{p} V(x) d x
$$

if and only if (1.3) is true; this strengthened version of Rosenblum's result follows from [2, Theorem 2, p. 215] since $\sup _{0 \leqslant r<1}\left|P_{r}(f, x)\right|$ is equivalent to the HardyLittlewood maximal function for nonnegative $f$. If the assumption $U(x)=V(x)$ is dropped, however, (1.7) does not imply (1.8); an example showing this is in $\S 5$ of [2]. In fact, the problem of characterizing the weight functions for which $(1.8)$ is true is unsolved and evidently quite difficult. Similarly, it is shown in [1] that if $1<p<\infty, U(x)$ and $V(x)$ are nonnegative and $U(x)=V(x)$, then (1.3) is necessary and sufficient for

$$
\int_{-\infty}^{\infty}|\tilde{f}(x)|^{p} U(x) d x \leqslant C \int_{-\infty}^{\infty}|f(x)|^{p} V(x) d x
$$

where $\tilde{f}(x)=\lim _{\epsilon \rightarrow 0^{+}}+\int_{|t|>\epsilon}(f(x-t) / t) d t$ is the Hilbert transform of $f(x)$. Again, (1.7) does not imply (1.9) if the assumption $U(x)=V(x)$ is dropped; for an example see [3]. It is rather surprising, therefore, that (1.7) is necessary and sufficient for (1.6) whether it is assumed that $U(x)=V(x)$ or not.

It should be noted that Theorem 1 does imply all of Rosenblum's result with his assumptions that $\mu=\nu$ and $\mu([-\pi, \pi])<\infty$. The only problem is to show that (1.5) implies that $\mu$ is absolutely continuous since (1.3) is obviously equivalent to (1.5) once absolute continuity is proved. To prove that $\mu$ is absolutely continuous, observe that since $\mu([-\pi, \pi])<\infty, d \mu_{a}(x) / d x<\infty$ almost everywhere. If $d \mu_{a}(x) / d x=0$ on a set of positive measure, then (1.5) implies that $\mu([-\pi, \pi])=0$ and $\mu$ is absolutely continuous. Therefore, assume that $0<$ $d \mu_{a}(x) / d x<\infty$ almost everywhere. Then, for any interval $I$,

$$
1 \leqslant\left(\frac{1}{|I|} \int_{I}\left[\frac{d \mu_{a}(x)}{d x}\right]^{1 / p}\left[\frac{d \mu_{a}(x)}{d x}\right]^{-1 / p} d x\right)^{p} .
$$

Applying Hölder's inequality to the right side of (1.10) then shows that

$$
1 \leqslant\left(\frac{\mu_{a}(I)}{|I|}\right)\left(\frac{1}{|I|} \int_{I}\left[\frac{d \mu_{a}(x)}{d x}\right]^{-1 /(p-1)} d x\right)^{p-1} .
$$

Multiplying (1.11) by $\mu(I)$ and using (1.5) shows that $\mu(I) \leqslant B \mu_{a}(I)$; since this is true for every interval $I, \mu$ is absolutely continuous.

2. Proof that (1.5) implies (1.4). The proof of this part of Theorem 1 will be done by proving two simple lemmas and then combining them. In this section and $\S 4$ the notation, $f_{h}(x)=h^{-1} \int_{x-h}^{x+h}|f(t)| d t$, will be used. 
LEMma 1. If $f(x)$ has period $2 \pi$ and $\mu$ and $\nu$ are Borel measures with period $2 \pi$ that satisfy (1.5), then for every $h>0$,

$$
\int_{-\pi}^{\pi}\left[f_{h}(x)\right]^{p} d \mu(x) \leqslant 6^{p+1} B \int_{-\pi}^{\pi}|f(t)|^{p} d \nu(x) .
$$

Assume first that $h \leqslant \pi$ and let $N$ be the least integer such that $N h \geqslant \dot{\pi}$. Then the left side of (2.1) is bounded by

$$
\sum_{k=-N}^{N-1} \int_{k h}^{(k+1) h} h^{-p}\left[\int_{x-h}^{x+h}|f(t)| d t\right]^{p} d \mu(x)
$$

and this is bounded by

$$
\sum_{k=-N}^{N-1}\left[h^{-p} \int_{k h}^{(k+1) h} d \mu(x)\right]\left[\int_{(k-1) h}^{(k+2) h}|f(t)| d t\right]^{p} .
$$

Using Hölder's inequality on the second integral in (2.2) shows that (2.2) is bounded by

$$
\begin{aligned}
& \sum_{k=-N}^{N-1}\left(\frac{\mu([k h,(k+1) h])}{h}\right)\left(\frac{1}{h} \int_{(k-1) h}^{(k+2) h}\right. {\left.\left[\frac{d \nu_{a}}{d x}\right]^{-1 /(p-1)} d x\right)^{p-1} } \\
& \cdot\left(\int_{(k-1) h}^{(k+2) h}|f(t)|^{p} d \nu_{a}(t)\right)
\end{aligned}
$$

note that if $d \nu_{a} / d x$ is 0 on a set of positive measure, $\mu([-\pi, \pi])=0$ and (2.2) is still bounded by this. Now (1.5) with $I=[(k-1) h,(k+2) h]$ shows that this is bounded by

$$
3^{p} B \sum_{k=-N}^{N-1} \int_{(k-1) h}^{(k+2) h}|f(t)|^{p} d v_{a}(t)
$$

Since all the intervals $[(k-1) h,(k+2) h]$ are subsets of $[-2 \pi, 2 \pi]$ and no point is in more than three of these intervals, $(2.3)$ is bounded by

$$
3^{p+1} 2 B \int_{-\pi}^{\pi}|f(t)|^{p} d \nu(t) .
$$

This completes the proof if $h \leqslant \pi$. If $h>\pi, f_{h}(x) \leqslant 2 f_{\pi}(x)$ and the result follows from the first part.

LEMMA 2. If $f(x)$ has period $2 \pi$ and $0 \leqslant r<1$, then there is a constant $K$, independent of $f$ and $r$, such that

$$
\left|P_{r}(f, x)\right| \leqslant K \int_{1-r}^{2 \pi}(1-r) h^{-2} f_{h}(x) d h .
$$

By reversing the order of integration, the integral on the right side of (2.4) is greater than or equal to 


$$
\int_{x \rightarrow \pi}^{x+\pi}|f(t)|\left[\int_{|x-t| \mathrm{V}(1-r)}^{2 \pi}(1-r) h^{-3} d h\right] d t
$$

where $|x-t| \vee(1-r)$ denotes the larger of $|x-t|$ and $1-r$. The inner integral can be calculated and is clearly greater than a positive constant times

$$
\frac{1}{2 \pi}\left(\frac{1-r^{2}}{(1-r)^{2}+2 r[1-\cos (x-t)]}\right)
$$

since $|x-t| \leqslant \pi$. This proves Lemma 2 .

To show that (1.5) implies (1.4), use Lemma 2 to show that the left side of (1.4) is bounded above by

$$
K^{p} \int_{-\pi}^{\pi}\left[\int_{1-r}^{2 \pi}(1-r) h^{-2} f_{h}(x) d x\right]^{p} d \mu(x)
$$

By Minkowski's integral inequality, (2.5) is bounded by

$$
K^{p}\left(\int_{1-r}^{2 \pi}\left[\int_{-\pi}^{\pi}\left[f_{h}(x)\right]^{p} d \mu(x)\right]^{1 / p}(1-r) h^{-2} d h\right)^{p}
$$

By Lemma 1, (2.6) is bounded by

$$
6^{p+1} B K^{p}\left[\int_{-\pi}^{\pi}|f(x)|^{p} d \nu(x)\right]\left[\int_{1-r}^{2 \pi}(1-r) h^{-2} d h\right]^{p}
$$

Since the last integral is less than $1,(1.4)$ follows.

3. Proof that (1.4) implies (1.5). This proof need only be done for intervals $I$ with $|I| \leqslant 2 \pi$, since if $|I|>2 \pi$ the left side of (1.5) is bounded by $2^{p}$ times its value for the interval $[-\pi, \pi]$. Given any $f(x)$, let $f_{1}(x)=0$ on the support of the singular part of $\nu$ and let $f_{1}(x)=f(x)$ elsewhere. Then $P_{r}\left(f_{1}, x\right)=P_{r}(f, x)$ and $\int_{-\pi}^{\pi}\left|f_{1}(x)\right|^{p} d \nu(x)=\int_{-\pi}^{\pi}|f(x)|^{p} d \nu_{a}(x)$. Therefore, if $d \nu_{a}(x) / d x$ is written as $V(x),(1.4)$ with $f$ replaced by $f_{1}$ implies that

$$
\int_{-\pi}^{\pi}\left|P_{r}(f, x)\right|^{p} d \mu(x) \leqslant C \int_{-\pi}^{\pi}|f(x)|^{p} V(x) d x .
$$

Consequently, the proof that (1.4) implies (1.5) can be completed by showing that (3.1) implies (1.5) for intervals $I$ with $|I| \leqslant 2 \pi$.

Given $I$ with $|I| \leqslant 2 \pi$, let $Q=\int_{I}[V(x)]^{-1 /(p-1)} d x$ and let $p^{\prime}=p /(p-1)$. If $Q=0,(1.5)$ follows because of the convention $0 \cdot \infty=0$. If $Q=\infty,[V(x)]^{-1 / p}$ is not in $L^{p^{\prime}}$ on $I$ so there is a function $g(x)$ in $L^{p}$ on $I$ such that $g(x)[V(x)]^{-1 / p}$ is not integrable on $I$. Let $f(x)=g(x)[V(x)]^{-1 / p}$ on $I$ and 0 elsewhere. Then $P_{r}(f, x)=\infty$ for all $x$ and the right side of (3.1) is finite since $g(x)$ is in $L^{p}$ on $I$. Therefore, $\mu(I)=0$ and (1.5) is true.

If $0<Q<\infty$ and $p>1$, let $f(x)=[V(x)]^{-1 /(p-1)}$ if $x+2 n \pi \in I$ for some integer $n$ and 0 elsewhere. Let $r$ be the larger of $1-|I|$ and 0 . Then for $x$ in $I$, 


$$
P_{r}(f, x) \geqslant \frac{A}{|I|} \int_{I}[V(y)]^{-1 /(p-1)} d y
$$

where $A$ is a positive constant independent of $x, V, p$ and $I$. Then (3.1) implies that

$$
\int_{I}\left[\frac{A}{|I|} \int_{I}[V(y)]^{-1 /(p-1)} d y\right]^{p} d \mu(x) \leqslant C \int_{I}[V(x)]^{-1 /(p-1)} d x .
$$

Dividing by the integral on the right side of (3.2) then gives (1.5).

If $0<Q<\infty$ and $p=1$, choose $\epsilon>0$ and let $E$ be the subset of $I$ where $V(x)<\epsilon+$ ess $\inf _{y \in I} V(y)$. Let $f(x)$ equal 1 if $x+2 n \pi \in E$ for some integer $n$ and 0 otherwise, and let $r$ be the larger of $1-|I|$ and 0 . Then for $x$ in $I, P_{r}(f, x)$ $\geqslant A|E| /|I|$ where $A$ is a positive constant independent of $x, V$ and $I$, and $|E|$ denotes the Lebesgue measure of $E$. Then (3.1) implies that

$$
\frac{A|E| \mu(D)}{|I|} \leqslant C|E|[\epsilon+\underset{y \in I}{\operatorname{ess} \inf } V(y)] \text {. }
$$

Dividing by $A|E|$ and using the fact that $\epsilon$ was arbitrary gives

$$
\frac{\mu(I)}{|I|} \leqslant \frac{C}{A} \text { ess } \inf _{y \in I} V(y) ;
$$

this is equivalent to (1.5) with the appropriate interpretation for $p=1$.

4. The nonperiodic case. Given $f(x)$ defined on $(-\infty, \infty)$, let

$$
f(t, x)=\frac{1}{\pi} \int_{-\infty}^{\infty} \frac{t f(y) d y}{t^{2}+(x-y)^{2}}
$$

be the usual Poisson integral. The nonperiodic theorem is the following.

THEOREM 2. If $1 \leqslant p<\infty, t>0$ and $\mu$ and $\nu$ are Borel measures, then there is a $C$, independent of $f$, such that

$$
\int_{-\infty}^{\infty}|f(t, x)|^{p} d \mu(x) \leqslant C \int_{-\infty}^{\infty}|f(x)|^{p} d \nu(x)
$$

if and only if for every interval I (1.5) holds where $B$ is independent of I and $\nu_{a}$ denotes the absolutely continuous part of $\nu$.

The proof that (1.5) implies (4.1) uses the following analogues of Lemmas 1 and 2.

LEMMA 3. If $\mu$ and $\nu$ are Borel measures that satisfy (1.5), then for every $h>0$

$$
\int_{-\infty}^{\infty}\left[f_{h}(x)\right]^{p} d \mu(x) \leqslant 3^{p+1} B \int_{-\infty}^{\infty}|f(t)|^{p} d \nu(x) .
$$

LEMMA 4. There is a constant $K$, independent of $f$ and $t$, such that $|f(t, x)| \leqslant K \int_{t}^{\infty} t^{-2} f_{h}(x) d h$. 
The proof of Lemma 3 is the same as that of Lemma 1 except that the sum is taken from $-\infty$ to $\infty$ and the initial restriction on the length of $I$ is not needed. Lemma 4 is proved in the same way that Lemma 2 was. The rest of the proof that (1.5) implies (4.1) is the same as the proof that (1.5) implies (1.4) except that Lemmas 3 and 4 are used in place of Lemmas 1 and 2.

The proof that (4.1) implies (1.5) is essentially the same as the proof in §3; the reduction to intervals $I$ with $|I| \leqslant 2 \pi$ is not needed and in the last two cases $t$ should be chosen equal to $|I|$ instead of $r$ being the larger of $1-|I|$ and 0 .

\section{REFERENCES}

1. R. Hunt, B. Muckenhoupt and R. L. Wheeden, Weighted norm inequalities for the conjugate function and Hilbert transform, Trans. Amer. Math. Soc. 176 (1973), 227-251. MR 47 \#701.

2. B. Muckenhoupt, Weighted norm inequalities for the Hardy maximal function, Trans. Amer. Math. Soc. 165 (1972), 207-226. MR 45 \#2461.

3. B. Muckenhoupt and R. L. Wheeden, Two weight function norm inequalities for the Hardy-Littlewood maximal function and the Hilbert transform, Studia Math. (to appear).

4. M. Rosenblum, Summability of Fourier series in $L^{p}(d \mu)$, Trans. Amer. Math. Soc. 105 (1962), 32-42. MR 28 \#3287.

DEPARTMENT OF MATHEMATICS, RUTGERS UNIVERSITY, NEW BRUNSWICK, NEW JERSEY 08903 